\title{
\%
}

Energy research Centre of the Netherland:

\section{Characterization of Li-ion Batteries for Intelligent Management of Distributed Grid Connected Storage}

\author{
J.D. Dogger \\ B. Roossien \\ F.D.J. Nieuwenhout
}

Published in IEEE Transactions on Energy Conversion, issue 99, 30 December 2010 


\title{
Characterization of Li-ion Batteries for Intelligent Management of Distributed Grid Connected Storage
}

\author{
Jarno D. Dogger, Bart Roossien, Member, IEEE, and Frans D.J. Nieuwenhout
}

\begin{abstract}
Grid connected electrical storage has a high potential to support the transition towards a reliable decentralized and renewable energy supply. It is expected that lithium-ion batteries will play a major role in this transition, because of their high energy density and of the potential capacity that is offered by plug-in (hybrid) electric vehicles. The use of lithiumion batteries in grid support may result in additional degradation. Intelligent control of these batteries can assure that the additional degradation rate is minimized and their utilization is costeffective. It is, therefore, imperative that the intelligent control has an excellent understanding of the aging behavior of the battery, so it can maximize the benefits for the battery owner. Based on this logic, cycle life experiments were performed on lithium polymer cells in which the cell life dependence on the depth of discharge was investigated. Other cell characteristics that were studied include the equivalent series resistance and the efficiency.
\end{abstract}

Index Terms-Lithium polymer, Cycle life, Depth of discharge, Equivalent series resistance

\section{INTRODUCTION}

With rising fuel prices and the call for reduction of carbon dioxide emissions, renewable and high efficiently electricity sources such as wind turbines, domestic combined heat and power generation and photo voltaic cells have become more popular than ever. Unlike conventional fossil-fuel power plants, most of these distributed electricity generators are not driven by the overall electricity demand but by external factors such as wind velocity, solar irradiation and the heat demand in buildings. Therefore, distributed generators might produce electricity at times when there is no electricity demand and visa verse, resulting in an imbalance between supply and demand. As long as the share of distributed generation (DG) in the electricity network is small, the conventional power plants can compensate for this imbalance. Future scenarios, however, show that distributed generation will most likely become the main source for electricity [1]-[3]. These large shares of DG will make it increasingly difficult for network operators to balance supply and demand of electricity. Eventually, this will lead to more unstable grids with an increased possibility of black outs as a result. A lot of research in solving these problems is focused on active management of electricity grids. Energy producing and consuming devices and appliances are intelligently controlled, utilizing their flexibility to counterbalance a deficiency or abundance in electricity. This principle has

Energy research Centre of the Netherlands (ECN), Power Systems and Information Technology, Westerduinweg 3, 1755LE Petten, The Netherlands Email: roossien@ecn.nl

Manuscript received September 28, 2008; revised May 29, 2009. been incorporated in a multi-agent based coordination concept named PowerMatcher [4], [5].

It has been demonstrated through simulations and fieldexperiments that such intelligent control of devices can coordinate the supply and demand when distributed generation provides the majority of the electricity in a grid [6], [7]. However, there are situations in which such a smart grid cannot maintain the power balance with flexible devices alone. In those situations, grid connected electrical storage systems can provide relief and any imbalance that cannot be compensated by intelligent control of devices is then exchanged with storage systems.

Central storage systems have little influence on local imbalances caused by DG and are also bound to power flow constraints in the network. By distributing many small grid connected storage systems over a wide area, local imbalances can be resolved, while the total storage capacity in the grid is still comparable to a central storage system. Only high energydensity batteries qualify for these distributed storage systems, because of their mobility, simplicity and flexibility. With the interest in plug-in (hybrid) electric vehicles (PHEV) growing by the day [8], distributed electrical storage in residential areas with batteries is already becoming a reality. Lithium-ion batteries seem to be the best candidate for this application, because of their potentially long cycle life, good deep cycling characteristics, high energy density, high efficiency and safe use. Also, many car manufacturers of hybrid and plug-in (hybrid) electric vehicles have opted for the use of lithiumion in the near future.

The challenge in controlling grid connected electrical storage is optimizing the financial benefits of the battery when used in multiple applications. One example is a PHEV battery offering ancillary services to increase the stability of the grid, an addition to its main function of supplying the car with electricity. Offering ancillary services to the grid increases the strain on the battery, thus reducing its life expectancy. One of the current issues with the use of lithium-ion batteries in grid connected applications is cycle life time. As lithium-ion cells gradually deteriorate under a cyclic load, irreversible chemical reactions causing an increase in cell resistance and decrease the number of available lithium ions, resulting in a reduced usable cell capacity. The aging rate and cycle life of the cell depends on many parameters such as charge and discharge rates, temperature, end of charge and discharge voltage and depth of discharge [9], [10]. This large list of parameters makes it difficult to predict the life expectancy of lithium-ion cells during operation, especially if the operating conditions are often changed. If the life expectancy of a battery can be 
predicted from its operational use, control strategies can be developed and incorporated in the intelligent control systems to prolong the life time of the battery by optimizing the participation in ancillary services together with the financial benefits. It can also provide network operators with a cost estimation of ancillary services.

In this paper, lithium-ion battery cycling experiments, specifically the application of grid connected storage in smart grids, is discussed, as well as which battery characteristics can be used most effectively to predict the cycle life of a battery in such application.

\section{EXPERIMENTS}

The most common way to study deterioration of lithium-ion batteries under cyclic loading is to determine the capacity fade by an $80 \%$ or $100 \%$ battery discharge. However, these tests were designed for an accelerated study of the battery lifetime. In real-life applications it is very rare that a battery undergoes such deep cyclic behavior but irregular charge/discharge patterns. For example, PHEV batteries are most likely fully charged at night and only partially discharged during the day. Therefore, our study is mainly focussed on the effects of the depth of discharge on the cycle life.

\section{A. Definitions}

In literature, slightly different definitions can be found for the state of charge and depth of discharge of a battery. To avoid confusion, this paragraphs will discuss the definition of these parameters as used in this article. The state of charge (SOC) of a battery is a measure of how much charge is left in that battery with respect to its nominal capacity, i.e.

$$
\text { SOC }=\frac{Q}{Q_{0}}
$$

where $Q$ is the amount of charge at a given moment and $Q_{0}$ is the nominal capacity of the battery. As the nominal capacity is a fixed value that does not change in time, aging has the effect that the SOC for a fully charged battery will slowly decrease in time.

The depth of discharge (DOD) is a measure of how much charge is drained from a battery in one cycle. To be exact, the DOD equals the change in the SOC in one discharge cycle, i.e.

$$
\mathrm{DOD}=\Delta \mathrm{SOC}=\frac{1}{Q_{0}} \int I(t) d t
$$

where $I$ the discharge current. A DOD of $60 \%$ means that the battery is discharged in its first cycle from a SOC of $100 \%$ until a SOC of $40 \%$. As battery aging takes place, the SOC of a fully charged cell after a number of cycles decrease to e.g. $97 \%$. The next discharge cycle with a DOD of $60 \%$ will then occur between a SOC of $97 \%$ and $37 \%$.

For the nominal battery capacity $Q_{0}$, we have chosen to use the name plate capacity (i.e. the capacity as specified by the manufacturer) instead of taking the actual capacity of a new battery. The latter is often determined by cycling a battery about 5 to 10 times before measuring its capacity and depends on the testing conditions. However, from an application point

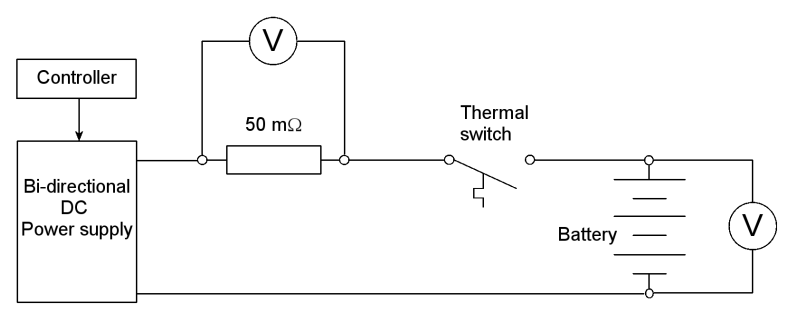

Fig. 1. Schematic outline of the battery test set-up

of view, it is only the name plate capacity of the battery that is known (specified by the manufacturer). It is also assumed that the batteries tested are equal in capacity and performance. Hence the use of the name plate capacity was chosen.

The charge / discharge current of a battery is usually expressed in terms of " $\mathrm{C}$ " and is equivalent to the inverse of the battery charging time (based on the nominal capacity), i.e.

$$
I=Q_{0} C
$$

For a battery with a nominal capacity of $1500 \mathrm{mAh}$, the charge current for respectively $1 \mathrm{C}$ and $\mathrm{C} / 10$ is $1.5 \mathrm{~A}$ and $0.15 \mathrm{~A}$.

\section{B. Set-up}

To allow for maximum flexibility, a custom-made battery test system was used as depicted in figure 1. Each test system contains a Kepco bipolar operational power supply BOP 36-6M, control unit, shunt and thermal switch. The bipolar power supply both charged and discharged the battery and was connected to the battery via the thermal switch, an additional safety measure to prevent the battery from overheating, although no such events have occured during testing. The power supply was regulated by the control unit, which was programmed to perform regular or irregular cycle patterns. The control unit also protected the lithium-ion cell from overloading or exceeding the lower voltage limit. The $50 \mathrm{~m} \Omega$ shunt was placed in the circuit between power supply and battery and was used to measure the current through the system. The wiring was kept as short as possible to minimize its resistance. Other measured quantities were the cell's terminal voltage and shell temperature as well as the ambient temperature. The system was situated in a climate controlled room where the ambient temperature was set to $25^{\circ} \mathrm{C}$. However, deviations from this set point have occurred during measurements due to incidental failure of the climate control system. An Agilent 34970A data acquisition unit was used to $\log$ the current, cell voltage, cell temperature and ambient temperature with a sample time of 1 minute.

The cells were charged using the well-known two step constant current constant voltage (CC-CV) protocol. First, a current of $1 \mathrm{C}$ was applied until the cell voltage limit of 4.2 volts approached. The system was switched to voltage control in which the voltage was held constant while the current gradually reduced. Charging was terminated when the current dropped below $\mathrm{C} / 20$. The use of such a relative high charge and discharge current has been chosen to mimick real life use in grid connected storage applications, such as charging a 
vehicle or absorbing a surplus production in wind energy. This way, the limits of the cells were tested. To reduce transient behavior in the chemistry, there was a 30 second break before discharging the cell, in which the current is zero.

A constant current of $1 \mathrm{C}$ was applied to discharge the cell. From measured current and time, the control unit calculated the amount of charge drained from the battery. The nameplate capacity has been entered manually in the control unit algorithm, so it could calculate the DOD at any time. When the DOD corresponded to the user-specified threshold, charging commenced and the cycle started again. Discharging the cell also ended when the cell voltage dropped below the cut-off voltage as specified by the manufacturer. The cut-off voltage (or lower limit voltage) averted the cell being too deeply discharged to avoid irreversible damage.

The cells were individually tested, i.e. each cell had its own test set-up as shown in figure 1 . In total 4 test set-ups were available, so that four cells could be tested individually at the same time.

\section{Cell testing}

At to the time of the preparation for the research as described in this article (2004), Lithium Polymer (LiPo) batteries were the most promising technology for grid connected electricity storage. In LiPo batteries, the lithium-salt electolyte is not held in an organic solvent, but in a solid polymer composite. With the absence of free-liquid, it can be packaged in light-weight cheap plastic containers. These "plastic" batteries are expected to be less expensive and easier to scale up than its liquid counterparts.

We have chosen to use the Kokam 1500mAh Superior Lithium Performance Batteries (SLPB) series SLB 603870H, which are LiPo batteries with a conventional cobalt oxide cathode. The manufacturer specifies a cycle life of at least 500 cycles (at $80 \%$ DOD), a lower cut-off voltage of 3.0 volts, a maximum charge current of $1 \mathrm{C}$ and a maximum discharge current of 5C. Larger versions of these Kokam SLPB cells are currently used in a variety of electric vehicles, like a Lotus Elise [11] or an electric racing car [12].

With the time needed to test a single cell (4 months up to 3 years per cell, depending on the test pattern) and with 4 test places available, only 10 cells were tested under partial discharge. Cells were discharged with $10 \%$ ( 1 cell), $20 \%$ (1 cell), $50 \%$ ( 2 cells), $80 \%$ ( 4 cells) and $90 \%$ ( 2 cells) of their name plate capacity. Two more cells have been tested in a capacity fade study, i.e. discharging the cell to its lower voltage limit in each cycle. The times to perform one partial cycle (discharge and charge) were 50 minutes (10\%), 87 minutes (20\%), 139 minutes (50\%) and 177 minutes (80\%). The time to perform one partial cycle remained constant up to the point where the battery failed. The definition of failure will be discussed in the next section.

\section{RESULTS}

\section{A. Cycling tests}

The discharge voltage characteristic at different cycle numbers for one of the two cells discharged with a depth of

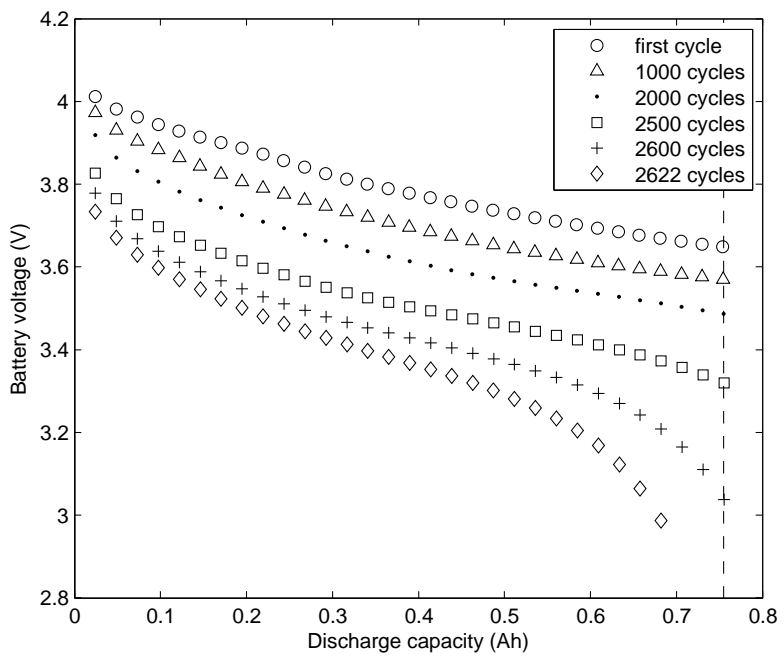

Fig. 2. Cell voltage versus the discharge capacity at different cycle numbers for a cell discharged with $50 \%$ of its name plate capacity and a current of $1.5 \mathrm{~A}(1 \mathrm{C})$

$50 \%$ is shown in figure 2. A few things can be observed, the voltage measured at the start of the discharge cycle is not only below the cell voltage limit of 4.2 volts, it also drops with increasing cycle number. The explanation can be found in the equivalent series resistance (ESR) of the cell. The open source voltage at this point is 4.2 volts, but because of the voltage drop across the ESR, the terminal voltage is lower. Cell deterioration increases the ESR resulting in a larger voltage drop and hence, a lower terminal voltage. The ESR will be discussed in more detail in section III-B.

Additionally, figure 2 also shows a decline in the end of discharge voltage, which is the voltage where cell discharge is stopped either because $50 \%$ of the nameplate capacity has been discharged or the lower cut-off limit of 3.0 volts is reached. The decline is caused by the increasing cell resistance and the loss of lithium ions in parasitic chemical reactions reducing the effective usable capacity of the cell. When the end of discharge voltage hits the lower voltage limit, the cell can no longer satisfy the imposed 50\% DOD specification and thus fails. In other words, the cell's cycle life is defined as the number of cycles that it can complete for a fixed DOD until it can no longer satisfy this discharge requirement. Failure for the cell in figure 2 occurs in cycle 2622. It is important to realize that our definition of cycle life is different from a more common definition, mainly used by battery manufacturers, which states that a cell fails when less than $80 \%$ of its initial capacity is left. This definition most likely find its roots in the expectation that below $80 \%$ the rate of degradation increases. As will be shown in section III-E, this definition might be flawed. However, our definition is based on a financial approach which is more reasonable for real-life applications. After all, replacing a battery that still has $80 \%$ of its capacity left is unnecessary, when only $50 \%$ is used in each cycle.

It is not possible to compare the number of cycles for batteries with different discharge depths directly, because of the unequal amounts of energy that flows through the system. A cycle of 10\% DOD exchanges only a fifth of the energy than 


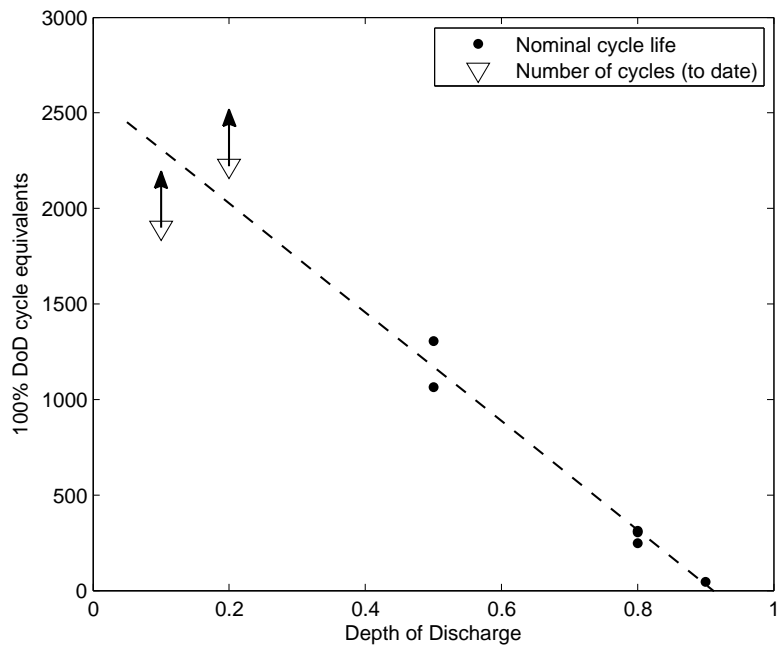

Fig. 3. The DOD plotted against the nominal cycle life. Note that the $10 \%$ and $20 \%$ cells have not reached failure yet.

one $50 \%$ DOD cycle. Multiplying the number of cycles with the DOD gives the nominal number of cycles, i.e. the number of $100 \%$ DOD cycle equivalents.

The nominal cycle life versus the DOD is represented in figure 3. Exceptions are the $10 \%$ and $20 \%$ DOD experiments, of which the number of cycles completed so far is shown, instead of their actual cycle life. The cell with a $10 \%$ DOD managed to complete almost 20,000 cycles (equivalent to 2,000 nominal cycles), before the experiment was aborted. The remaining capacity in the cell at that moment was still $74 \%$ of its name-plate capacity. The $20 \%$ DOD cell is still running after three years while already having performed over 10,000 partial cycles. Its final results will be published in future work. If a straight line is drawn through all points, it would cut the $\mathrm{x}$ axis at DOD $=0.93$. However, it is expected that the line cuts the $\mathrm{x}$-axis at $\mathrm{DOD}=1$. This observation follows from using the name-plate capacity as the reference capacity instead of the real cell capacity for the described test procedure. It can, therefore, be concluded that on average the usable capacity of a new cell in our test conditions is $93 \%$ of the name plate capacity.

Figure 3 shows that the lower the DOD of a cell, the longer its nominal cycle life is. Therefore, more energy is exchanged during a cell's life time if a cell has a low DOD. The total energy that flows through a 50\% and $80 \%$ discharged cell during its lifetime was found to be $7.3 \pm 0.2 \mathrm{kWh}$ and $1.5 \pm 0.2 \mathrm{kWh}$ respectively. There are two reasons that explain this characteristic. First, a cell is allowed greater amounts of capacity loss when it has a low DOD, which follows from the definition of cell "failure". A cell that is discharged with $50 \%$ can lose $50 \%$ of its capacity, while a cell discharged with $80 \%$ can lose only $20 \%$ of its capacity. Secondly, the strain on the battery is less with a lower DOD as less lithium ions react in each cycle. This results in a lower capacity loss rate, increasing the cycle life time of the cell. The amount of energy that flows through the battery during its life time is an important characteristic for the economical feasibility of grid connected storage applications. Assuming a fixed profit per unit of energy

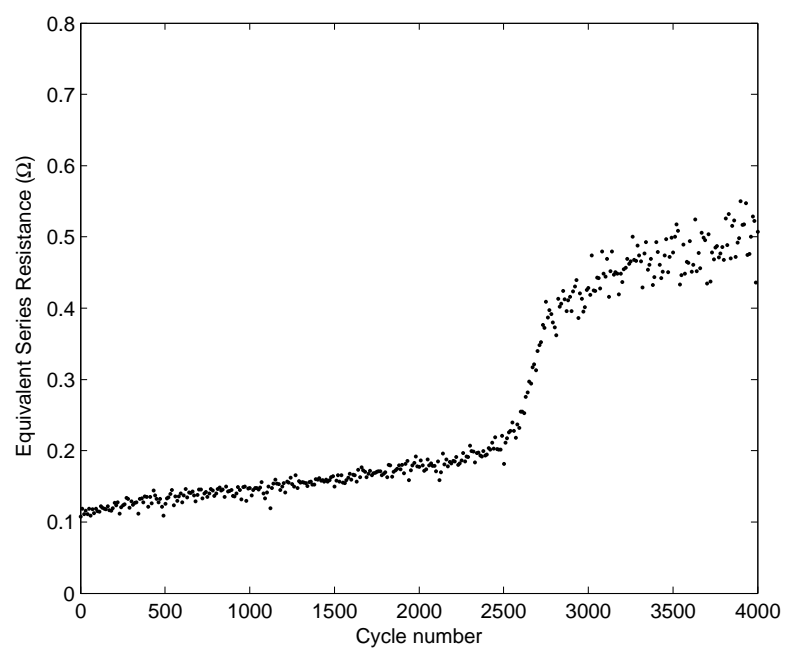

Fig. 4. Equivalent series resistance plotted against the cycle number after temperature correction (see figure 6) for a cell discharged with $50 \%$ of its name plate capacity.

that flows through the cell, the $50 \%$ discharged cell generates almost 5 times more income than the $80 \%$ discharged cell during its life time.

A relation between the nominal cycle life and the depth of discharge cannot yet be deducted from figure 3 because of the limited number of data points available. For DOD $>0.5$ the relation is approximately linear, but it appears as though the nominal cycle life for small $(<0.5)$ DoDs can be found above this imaginary straight line. Hence the relation between DOD and nominal cycle life is probably not linear.

\section{B. Equivalent series resistance}

Already mentioned in the previous section is the equivalent series resistance (ESR). In the performed experiments, the ESR was found by measuring the voltage drop at the current step between charging ( $I=0 \mathrm{~A})$ and discharging $(I=-1.5$ A) the cell. As voltage measurements are done at fixed time steps, it was not possible to measure the exact voltage directly after the current step. Instead, linear interpolation was used to obtain the voltage drop which resulted in a 10-cycle average value of the ESR at charged state.

The ESR for a cell discharged with a depth of $50 \%$ is plotted as function of its cycle number in figure 4 after temperature correction (see section III-C). Three phases can be distinguished in its behavior. In the first phase, $(0 \leq$ cycles $<2500)$ the cell is discharged with a constant DOD. The transition to the second phase $(2500<$ cycles $<2800)$ is situated at the point of cell failure. The ESR's rate of change in the second phase is higher than in the first phase and can be attributed to the absolute discharge depth of the cell. In cycles $<2500$, the cell is not entirely discharged, opposite to cycles $>2500$. It is well known that a deeper discharge causes increases the deterioration rate of the cell [13], resulting in a faster increase of the ESR. In the third phase (cycles > 2800) however, the ESR rate decreases again. It is believed to be caused by the lack of lithium-ions that participate in parasitic side reactions, as most have been consumed. As a result, even the 


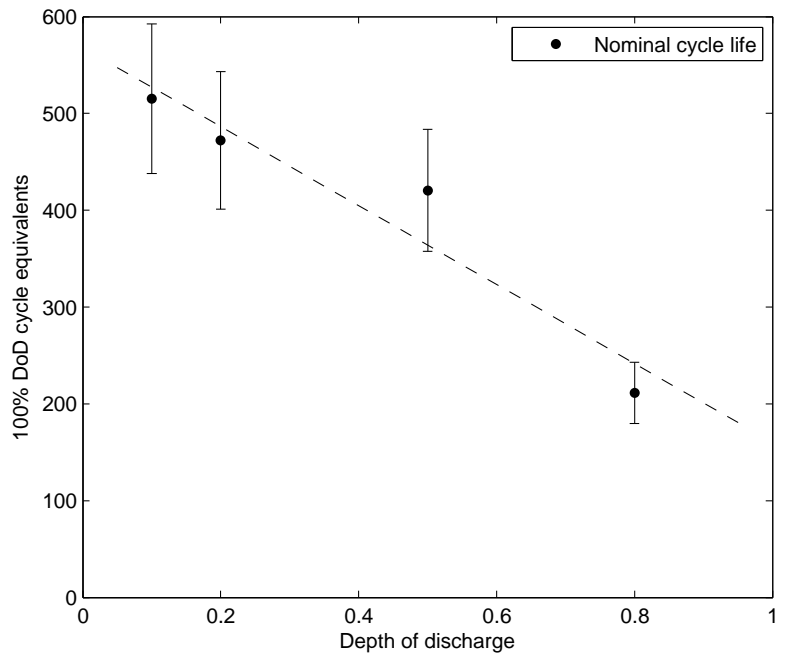

Fig. 5. Nominal cycle life that has been performed before the ESR increases to 1.3 times its initial value for cells discharged with different depths of discharge.

parasitic chemical reactions occur at a slower rate. In the third phase, it was measured that the remaining capacity of the cell comprised of less than $10 \%$ of its nominal capacity. The main interests, however, were focussed on the first phase, which is the normal operational area of the cell.

The increase of the ESR during the lifetime of a cell has a major impact on the trading behaviour for grid connected storage. Storage that is solely used to trade electricity (e.g. on a power exchange market) sells its electricity against a higher price than the price for which it bought in order to make a profit. As energy is lost between buying and selling (i.e. charging and discharging), the sell price does have to include the costs for these losses. Therefore, the minimum sell price $p_{\text {sell }}$ can be expressed in terms of the buy price $p_{b u y}$ and the cycle efficiency $\eta$ of the storage system following

$$
p_{\text {sell }} \geq p_{\text {buy }} / \eta
$$

As the ESR increases, the cycle efficiency decreases and the gap between the buy and sell price increases. This makes the storage system less competitive in the market.

The initial value for the ESR when new was found to be $R_{0}=0.12 \pm 0.01 \Omega$ for all measured cells. A less common, but not unusual, definition of the battery cycle life is based on the ESR and is defined as the number of cycles the battery can perform before its ESR increases to 1.3 times its initial value. The relation between the DOD and the nominal cycle life based on this definition is shown in figure 5. As opposed to figure 3 , where the number of cycles completed to date are shown for the $10 \%$ and 20\% DOD cells, here they are shown with their actual cycle life. As the figure shows, more nominal cycles can be performed when a cell is discharged with a lower DOD. A linear relation between the DOD and the nominal cycle life can be detected, but whether this is a one time result has to be studied in future experiments. Nonetheless, from a grid connected storage perspective, it is desired to have shallow depths to keep the increase rate of the ESR low. This will lengthen the storage system competitiveness in the trade

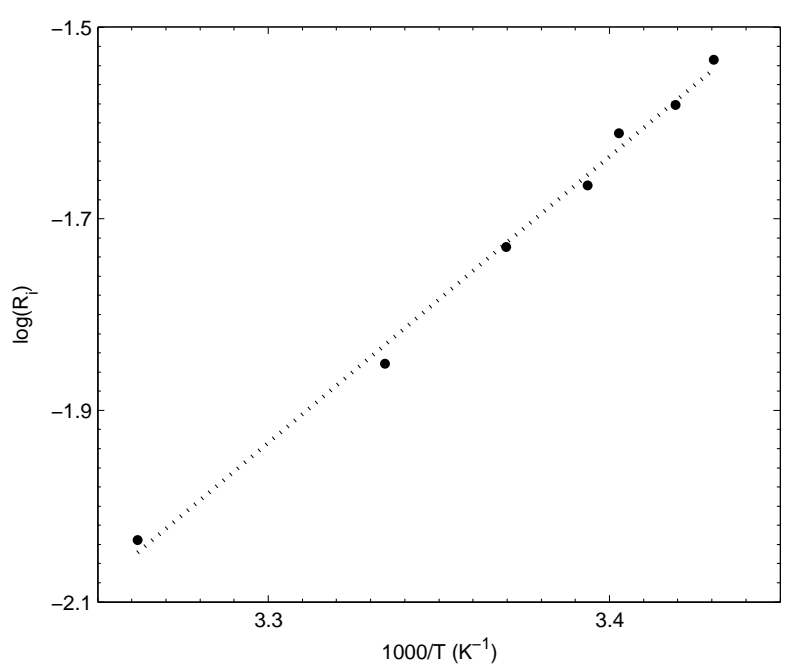

Fig. 6. Temperature dependence of the equivalent series resistance

market.

\section{Temperature vs ESR}

In a period of 5 days, variations in ambient temperature have been observed due to air conditioning failures in the peak summer period. Although data is limited to these 5 days (i.e. 40-100 cycles) it has allowed us to study the relation between the ESR and ambient temperature. Due to the relatively short time scale in which the temperature variations occurred, the increase in ESR resulting from aging was neglected. The relation between ESR and temperature for a cell with a $50 \%$ DOD is shown in the Arrhenius-type plot in figure 6, where the logarithmic ESR is displayed as function of the inverse of the ambient temperature. The ESR increases rapidly with decreasing temperature and may be caused by the lower ionic conductivity in the electrode and polymer gel and/or slower chemical reactions in the cell. This exponential behavior of the ESR, as function of temperature, corresponds with earlier found results in literature [14]. A straight line fitted through the data points resulted in the following relation between the ESR $R$ and temperature $T$

$$
R=R_{0} e^{A / T}
$$

where $R_{0}$ is the temperature independent and cell specific ESR and $A$ is the Arrhenius relational parameter. All four cells that have been tested in this period shows this Arrhenius relation. The average value and standard deviation of the parameter $A$ for these cells was found to be $3.1 \pm 0.2 \cdot 10^{3} \mathrm{~K}$.

The dependence of the ESR on temperature is important for grid connected storage systems that are not located in a conditioned space, e.g. plug-in (hybrid) electric vehicles or substations with electricity storage. From a trading perspective, the sell price for the stored electricity must be higher than the buy price plus the costs for losses. As temperature changes, the ESR changes and with that, the costs for losses. Hence, there exists a relation between the ambient temperature and the relative sell price with respect to the buy price of electricity. 

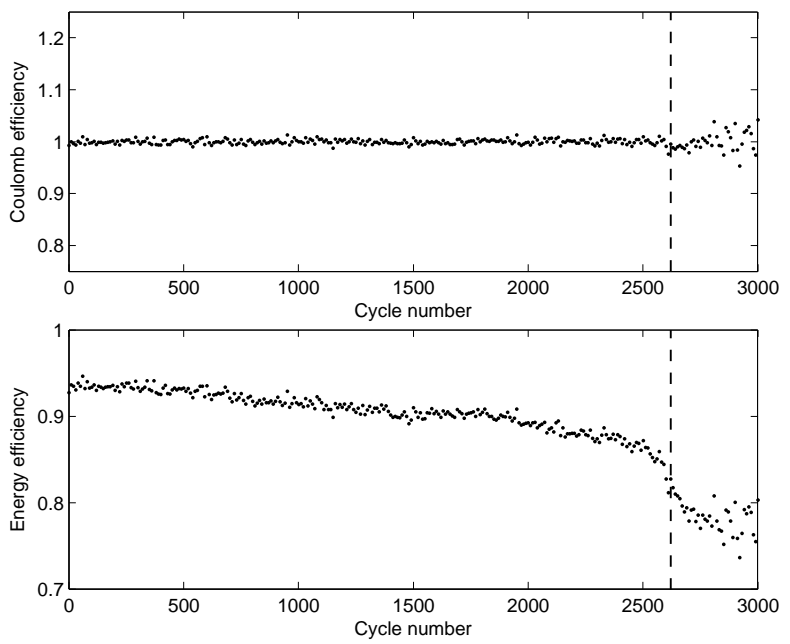

Fig. 7. Coulomb (top) and energy (bottom) efficiency for a cell discharged with $50 \%$ of its name plate capacity. The vertical dashed lines denote the point of failure of the cell.

\section{Efficiency and ESR}

Another important characteristic of lithium-ion cells is the efficiency. The Coulomb efficiency $\eta_{c}$ and energy efficiency $\eta_{e}$ are per definition the ratio between charge, respectively energy output and input in one cycle, i.e.

$$
\begin{gathered}
\eta_{c}=\frac{Q_{\text {discharge }}}{Q_{\text {charge }}}=\frac{\int_{d} I(t) d t}{\int_{c} I(t) d t} \\
\eta_{e}=\frac{E_{\text {discharge }}}{E_{\text {charge }}}=\frac{\int_{d} V(t) I(t) d t}{\int_{c} V(t) I(t) d t}
\end{gathered}
$$

where $I(t)$ and $V(t)$ are the measured respective current and voltage and $\int_{c}$ and $\int_{d}$ the time integrals over the charge and discharge periods in one cycle. Figure 7 shows the 10-cycle average coulomb and energy efficiency for a cell discharged with a depth of $50 \%$ of its name plate capacity. Variations in the efficiency are a result of the sample time resolution. The coulomb efficiency is approximately one throughout the experiment, so it is not being affected by the aging of the cell. The opposite is true for the energy efficiency. The efficiency gradually reduced with increasing cycle numbers. In the first few cycles, the energy efficiency was found to be $\eta_{e}=0.94 \pm 0.01$ for all tested cells which is not an uncommon value for lithium-ion batteries [15]. As failure was approached (denoted by the dashed line), the energy efficiency reduced to $\eta_{e}=0.85 \pm 0.01$. It is believed that this drop in energy efficiency can be contributed to the increase of the equivalent series resistance, as shown in figure 4.

Assuming the cycle energy loss is caused by the ESR only and not by any chemical side-reactions, the energy that flows into the battery in each cycle equals the outgoing flow minus resistance losses, i.e.

$$
E_{\text {discharge }}=E_{\text {charge }}-R_{i} \int_{T} I^{2}(t) d t
$$

with $R_{i}$ the ESR and $T$ the cycle time period. Substituting

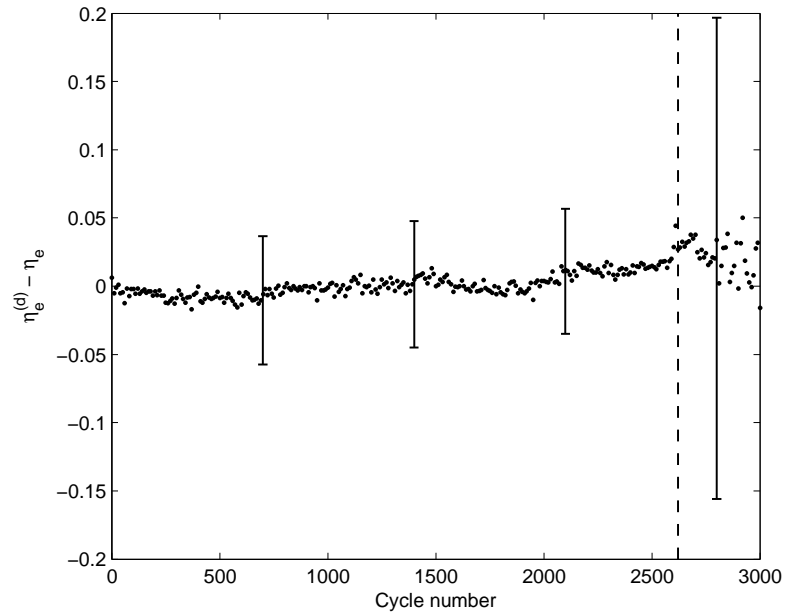

Fig. 8. Deviation between measured and calculated energy efficiency

equation (8) in (7), one obtains:

$$
\eta_{e}^{(d)}=\left[1+\frac{R_{i}}{E_{\text {discharge }}} \int_{T} I^{2}(t) d t\right]^{-1}
$$

in which the dependence on the energy input from charging has been eliminated. The absolute difference between $\eta_{e}^{(d)}$ and $\eta_{e}$ is plotted in figure 8 . The error bar is based on the measurement variations caused by the time step resolution. From figure 8 it is immediately clear that any difference in efficiency can be neglected with regard to the size of the error. In other words, $\eta_{e}^{(d)}$ and $\eta_{e}$ are equal. This makes the earlier made assumption about the ESR being responsible for all energy loss plausible. However, one expects the ESR to vary with the SOC [16], [17] and only the ESR at charged state was measured in this experiment. Future research will have to show whether the influence of the SOC on the ESR is significant for this type of cell and if so, what other parameters play a part in the cell's energy efficiency. Even if the energy loss might not be entirely contributed to the ESR, the ESR remains an important parameter in competitive market trading. The ESR is fairly easy to measure as opposed to the cycle efficiency, because of the irregular charge/discharge patterns and currents used in real applications.

\section{E. Capacity fade}

Finally a capacity fade experiment was performed as a worst case scenario for a lithium-ion cell. A capacity fade study discharges the cell in each cycle to the lower voltage limit (3.0 volts) with the same charge and discharge rates as described in the beginning of this article. The result is displayed in figure 9 where the relative remaining capacity $Q / Q_{0}$ is initially 0.93 instead of 1 . As explained in section III-A, the actual capacity of a new cell is usually lower than the name plate capacity, while the latter was used as reference. As expected, the initial capacity value of 0.93 for a cell corresponds with the value where the line in figure 3 crosses the $\mathrm{x}$-axis. It can be concluded that all cells under the described test conditions have an actual capacity that is $0.93 \pm 0.01$ times the name plate capacity, which justifies the assumption made in section 


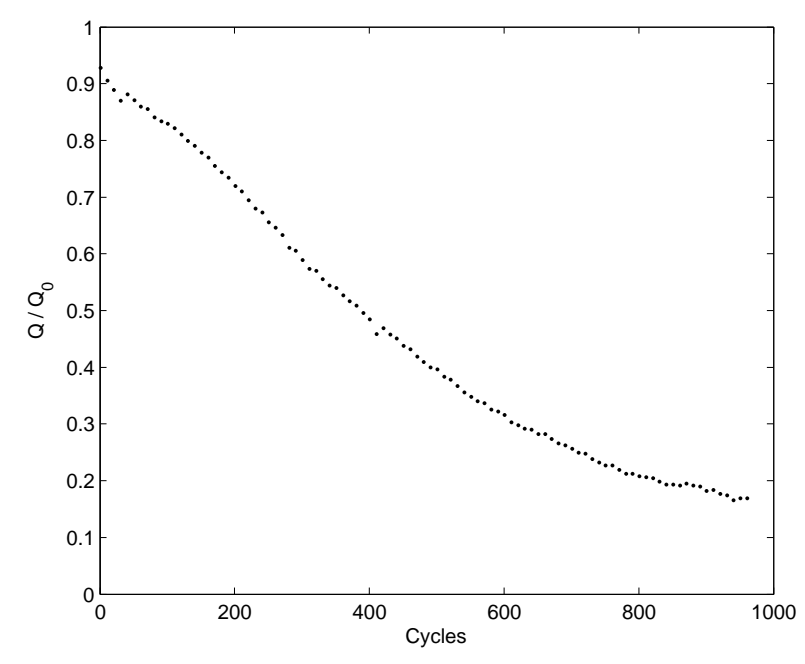

Fig. 9. Remaining capacity as function of the number of cycles

II-A; that all tested batteries can be considered equal. The difference between name plate capacity and actual capacity can be contributed to the test cycle that was used to measure the capacity. The manufacturer has likely used a different, lessaggressive test cycle.

Figure 9 is characterized by the sharp decrease of the remaining capacity of the cell. It took less than 200 cycles to reduce the capacity by $20 \%$, and after about 1000 cycles, less than $15 \%$ of the capacity still remained in the cell. With such a high degradation, it is obvious that these cells are very unsuitable for deep discharge cycling. Although the rate in capacity reduction is high, it does not show significant changes over time. Hence, it is questioned whether the definition of battery failure used by manufacturers $(\leq 80 \%$ remaining capacity) is really justified. It might be something that can only be applied on certain types of batteries. A more rigid definition, as was proposed, is more suitable for real-life applications of batteries, especially if the batteries are only subject to partial cycles.

\section{DISCUSSION}

From the presented results, it is clear that the cells have a lower degredation rate if they are subject to partial discharge cycles only. Although the flow of energy in each cycle is lower with respect to full discharge cycles, the total energy flow during the life time of the battery is higher. It is, therefore, interesting to look at the different options for grid connected storage from a financial point of view. A single battery that is $80 \%$ discharged in each cycle exhanges the same amount of energy as two identical batteries that are only $40 \%$ discharged. Thus the total cycle capacity of the storage system does not change, but the battery degradation rate in the single-battery storage system is higher for three reasons. First, because of the larger DOD, the degradation rate is higher. Secondly, more capacity can be lost before the battery can no longer fullfill its requirements. Finally, with two batteries, the current needed for the same application is only half of that of a single battery. It is well known that lower currents reduce the degradation rate of the battery. The result is a longer life time, which allows for higher profits.

However, it should be considered that not all applications can replace a single battery by two partial discharged batteries. First, there is the investement costs. Although it is economical more favorable to have two batteries, the intitial costs of already highly priced lithium-ion batteries can be a barrier. Secondly, other characteristics like weight or volume can play their part. For a car, the weight is limited. With the current lithium-ion technology, about $1 \mathrm{~kg}$ of batteries is needed for every kilometer of range the car needs to have. For storage in homes or (existing) substations, the volume can be a bottleneck.

One of the things not studied was the effect at the charged side of the battery. The degradation of battery that is completely emptied in every cycle is higher than for a battery which is only partial emptied. This also holds for a battery that is completely charged. A battery that is not completely charged, but e.g. charge to a lower end of charge voltage (EOCV) like 3.9 or 4.0 volts instead of 4.2 volts, experiences less degradation [18]. Furthermore, the capacity fade of the partial cycled batteries was not analyzed due to insufficient data. It is recommended, for future work, that at regular cycle intervals during testing the capacity is measured, although the additional degradations following from this measurement might disturb the results.

\section{CONCLUSIONS AND FUTURE WORK}

In this article, the importance of the role that batteries will play in future smart grids has been discussed. However, intelligent control of these batteries in e.g. ancillary services for the grid, requires knowledge about the aging behavior of the battery depending on its use. This way, the intelligent algorithm can optimize the technical and financial benefits of the battery. In this article, it was shown that a number of points are vital for an aging prediction model for real-life applications. The depth of discharge (DOD) has a significant effect on the cycle life of a battery. The smaller the DOD, the longer the cycle life and the more energy it can store during its lifetime. For real applications, the DOD of discharge varies with each cycle and the aging behavior of lithium-ion cells at irregular discharge patterns will be studied in future experiments.

The behavior of the equivalent series resistance (ESR) has also been discussed. The closer the end of discharge voltage approaches, the faster the ESR increases. Additionally, the rate at which the ESR increases depends on the DOD. The ESR increases faster when the cell makes deep discharge cycles. Furthermore, the ESR and the energy efficiency may be correlated. Based on these observations, it was concluded that the ESR is the most suitable parameter that can be measured within an operating storage system to predict the cycle life of the battery, using its historic loads. Future experiments and simulations have to show how the ESR can predict the cycle life of a cell accurately, and if this relation also holds for other manufacturers and types of lithium-ion batteries. 


\section{REFERENCES}

[1] M. ten Donkelaar, "A survey of solutions and options for the integration of distributed generation into electricity supply systems," Energy and Environment, vol. 15(2), pp. 323-332, 2004.

[2] D. Coll-Mayor, M. Paget, and E. Lightner, "Future intelligent power grids," Energy Policy, vol. 35, pp. 2453-2465, 2007.

[3] "European smartgrids technology platform : Vision and strategy for europe's electricity network of the future," 2006, iSBN:9279014145.

[4] M. Hommelberg, C. Warmer, I. Kamphuis, J. Kok, and G. Schaeffer, "Distributed control concepts using multi-agent technology and automatic markets," in Proceedings of the IEEE PES annual conference, 24-28 June, 2007.

[5] The PowerMatcher has its own website: http://www.powermatcher.net.

[6] C. Warmer, M. Hommelberg, B. Roossien, J. Kok, and J. Turkstra, "A field test using agents for coordination of residential micro-chp," in Proceedings of the 14th International Conference on Intelligent System Applications to Power Systems (ISAP), November 4 - 8, 2007.

[7] B. Roossien, "Field test upscaling of multi-agent coordination in the electricity grid," in Proceedings of the 20th CIRED conference, 8-11 June, Prague, 2009.

[8] J. Romm, "The car and fuel of the future," Energy Policy, vol. 34, pp. 2609-2614, 2006

[9] P. Ramadass, B. Haran, R. White, and B. Popov, "Performance study of commercial licoo2 and spinel-based li-ion cells," J. Power Sources, vol. 111, pp. 210-220, 2002.

[10] P. Arora, R. White, and M. Doyle, "Capacity fade mechanisms and side reactions in lithium-ion batteries," J. Electrochem. Soc., vol. 145(10), p. 3627, 1998.

[11] Electric Car Europe, http://www.ececars.nl.

[12] ProEV, http://www.proev.com.

[13] W. van Schalwijk and B. Scrosati, Advances in Lithium Batteries. ISBN 0306473569, 2002, chapter 13

[14] B. Ratnakumar, M. Smart, L. Whitcanack, and R. Ewell, "The impedance characteristics of mars exploration rover li-ion batteries," $J$. Power Sources, vol. 159, pp. 1428-1439, 2006.

[15] B. Kuhn, G. Pitel, and P. Krein, "Electrical properties and equalization of lithium-ion cells in automotive applications," Proceedings of the 2005 IEEE vehicle power and propulsion conference, pp. 55-59, 2005.

[16] I. Bloom, B. Cole, J. Sohn, S. Jones, E. Polzin, V. Battaglia, G. Henriksen, C. Motloch, R. Richardson, T. Unkelhaeuser, D. Ingersoll, and H. Case, "An accelerated calendar and cycle life study of li-ion cells," J. Power Sources, vol. 101, pp. 238-247, 2001.

[17] J. Shim and K. Striebel, "Characterization of high-power lithium-ion cells during constant current cycling," J. Power Sources, vol. 122, pp. 188-194, 2003.

[18] P. Ramadass, B. Haran, P. Gomadam, R. White, and B. Popov, "Development of first principles capacity fade model for li-ion cells," $J$. Electrochem. Soc., vol. 151 (2), pp. A196-A203, 2004.

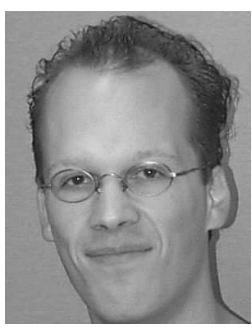

Jarno D. Dogger was born near Amsterdam in the Netherlands in 1972. He received a BSc degree in control engineering and electrical energy conversion from the Alkmaar university of professional education, The Netherlands, in 1996

$\mathrm{He}$ has been working at the Energy research Centre of the Netherlands since 1999, first on solar energy applications while specializing on electric power conversion, and later on electricity storage management systems. His current focus is grid support with grid connected (storage) systems and plug-

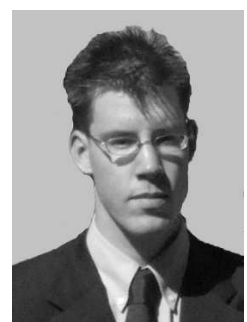

simulations.

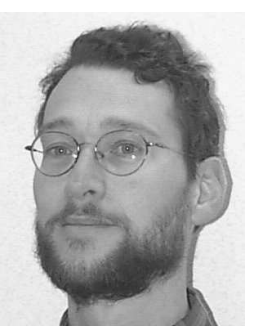

Bart Roossien (M'08) was born in Groningen, the Netherlands in 1983. He received a MSc degree in applied physics from the University of Groningen, The Netherlands, in 2006.

$\mathrm{He}$ is currently working at the Energy research Centre of the Netherlands, where he works on the topic of intelligent energy grids and grid connected electrical storage. His main topics of interests are: multi-agent technology, intelligent control of devices, the prediction of property behavior and time series and the development of computer models and

Frans D.J. Nieuwenhout was born near Rotterdam in the Netherlands in 1957 . He received a BSc degree in Astronomy and a MSc degree in experimental physics from the Utrecht University, The Netherlands in 1985.

$\mathrm{He}$ has been working at the Energy research Centre of the Netherlands since 1990, first on energy policy issues in developing countries, and later on solar energy applications. His current focus is on smart grids and electricity storage.

in electric vehicles. 